\title{
Learning curve for port-access thoracoscopic anatomic lung segmentectomy
}

\author{
Akira Hamada, MD, ${ }^{a}$ Hiroyuki Oizumi, MD, PhD, ${ }^{a}$ Hirohisa Kato, MD, PhD, ${ }^{a}$ Jun Suzuki, MD, \\ Kenta Nakahashi, MD, ${ }^{a}$ Ri Sho, MD, PhD, ${ }^{b}$ and Mitsuaki Sadahiro, MD, PhD ${ }^{a}$
}

\section{ABSTRACT}

Objectives: There have been few prospective randomized studies, but many retrospective studies strongly suggest the benefits of segmentectomy in properly selected patients. The indications for video-assisted thoracic surgery segmentectomy are growing because of the effectiveness and minimal invasiveness of the procedure. The aim of the present study was to analyze the learning curve for video-assisted thoracic surgery segmentectomy procedures in our institution.

Methods: We prospectively collected data from patients undergoing videoassisted thoracic surgery segmentectomy and retrospectively reviewed 252 patients from 2004 to 2015. Operative time, bleeding, and complications were analyzed. The learning curve was evaluated using operative time and the cumulative sum value of operative time in all cases with regard to the leading surgeon and nonleading surgeon at our institution.

Results: Once we applied the cumulative sum method to all cases, we obtained a graph for the cumulative sum value of operative time that showed 3 welldifferentiated phases: phase $1(n=61)$, the initial learning phase; phase 2 $(n=23)$, the increased competence phase; and phase $3(n=168)$, the highest skill phase. As we compared phases 1 and 2 with phase 3 , we observed significant differences in relation to operative time $(P<.001)$ and bleeding $(P<.001)$. Without level 3 segmentectomy, we observed a significant reduction in operative time after 32 cases for the leading surgeon and a significant reduction in operative time and bleeding after 38 cases for the nonleading surgeon.

Conclusions: The data suggest that the inflection point for the learning curve was achieved after 84 cases in our institution. Therefore, increased aptitude with video-assisted thoracic surgery is achievable within a relatively short time. (J Thorac Cardiovasc Surg 2018;156:1995-2003)

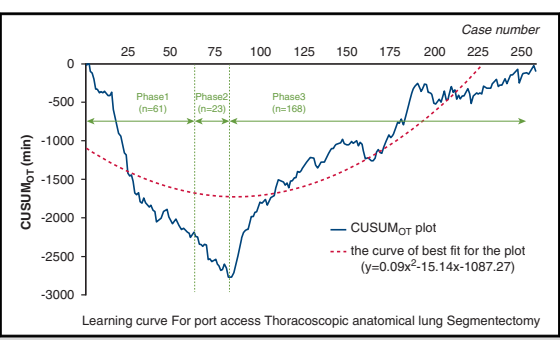

Learning curve for a port-access thoracoscopic anatomic lung segmentectomy.

Central Message

Increased aptitude, indicated by the inflection point of the learning curve for port-access thoracoscopic anatomic lung segmentectomy, is achievable in a relatively short time with VATS

\section{Perspective}

Segmentectomy is considered likely to become a standard surgical technique in the near future because of its superiority for postoperative pulmonary function and noninferiority with regard to overall survival for early-stage lung cancer. VATS segmentectomy is difficult to perform but is minimally invasive and will become a necessary procedure in thoracic surgery.

See Editorial Commentary page 2004.
Video-assisted thoracic surgery (VATS) lobectomy with systematic node dissection for non-small cell lung cancer has been used with increasing frequency since it was first performed in 1995 by McKenna. ${ }^{1}$ Studies have provided strong evidence that the VATS lobectomy is an acceptable alternative to an open lobectomy for treating early-stage

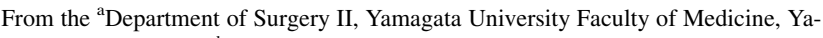
magata, Japan; and ${ }^{\mathrm{b}}$ Department of Public Health and Hygiene, Yamagata University Graduate School of Medical Science, Yamagata, Japan.

Institutional Review Board approval No. 2017-484 (Dated: January 29, 2018.)

Received for publication Dec 25, 2017; revisions received June 7, 2018; accepted for publication June 12, 2018; available ahead of print Aug 16, 2018.

Address for reprints: Hiroyuki Oizumi, MD, PhD, Department of Surgery II, Yamagata University Faculty of Medicine, 2-2-2 Iida-Nishi, Yamagata 990-9585, Japan (E-mail: hohizumi@med.id.yamagata-u.ac.jp).

$0022-5223 / \$ 36.00$

Copyright (c) 2018 by The American Association for Thoracic Surgery

https://doi.org/10.1016/j.jtcvs.2018.06.082
}

non-small cell lung cancer. $^{2-5}$ There are little data regarding the benefits of the VATS segmentectomy; however, indications for the use of VATS segmentectomy are recently becoming more widespread because of the effectiveness and minimal invasiveness of the procedure. We have experience with more than 250 cases of VATS anatomic segmentectomies at our institution. We have previously reported the use of 3-dimensional (3D) multidetector computed tomography (CT) images for surgical simulation during an anatomic thoracoscopic lung

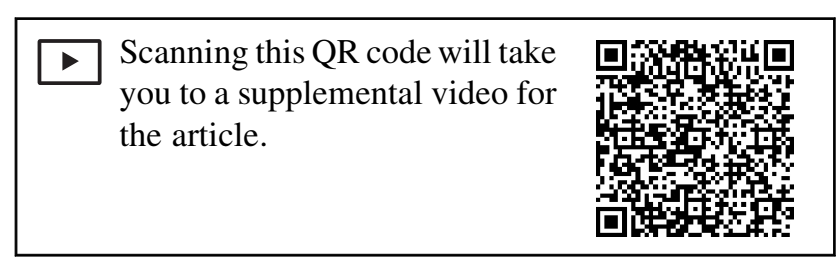




$$
\begin{aligned}
& \text { Abbreviations and Acronyms } \\
& \text { ASA }=\text { American Society of } \\
& \text { Anesthesiologists } \\
& \text { CT = computed tomography } \\
& \text { CUSUM = cumulative sum } \\
& \text { CUSUM }_{\mathrm{OT}}=\text { cumulative sum value of operative } \\
& \text { time } \\
& \text { LOS = length of stay } \\
& \text { OT }=\text { operative time } \\
& 3 \mathrm{D} \quad=3 \text {-dimensional } \\
& \text { VATS }=\text { video-assisted thoracic surgery }
\end{aligned}
$$

segmentectomy and the effectiveness of this technique. ${ }^{6,7}$ Furthermore, we also reported the slip-knot bronchial ligation method for visualization of the anatomic plane and inflation-deflation line during lung segmentectomy, as well as other alternative methods. ${ }^{8-11}$ These techniques facilitated the performance of the port-access anatomic lung segmentectomy. To standardize these techniques, we considered that analyzing the learning curve is important. The aim of the present study was to analyze the learning curve for VATS segmentectomy in our institution. Also, because this is an educational hospital, the leading surgeon teaches these techniques to residents, and gradually the residents master these techniques. Learning curves are different between the leading surgeon and the nonleading surgeon; therefore, we analyzed the learning curve of each.

\section{MATERIALS AND METHODS}

We prospectively collected the data from patients receiving this surgery and retrospectively reviewed the data from 252 consecutive patients who underwent a VATS segmentectomy from September 2004 to December 2015. During this study period, we had 204 cases of VATS lobectomy, 242 cases of open lobectomy, and 305 cases of segmentectomy. The clinical data analyzed for each case included age, sex, operative side, American Society of Anesthesiologists (ASA) score, comorbidity index, surgical side, segmentectomy level, operative time (OT), bleeding, days of drainage, length of stay (LOS) in hospital, complications, 30-day mortality rate, 30day readmission rate, and pathologic diagnosis. All procedures were controlled on the monitor, and there was no use of a rib spreader. In each case, branches were identified using 3D CT angiography imaging. The segmentectomy was performed using the slip-knot bronchial ligation method for visualization of the anatomic plane and the inflation-deflation line during the lung segmentectomy. The polymer clip was used as an alternative method to close the bronchial stump during a subsegmentectomy. The posterior approach was performed for segmentectomy of the dorsal basal segment. We performed lymph node sampling instead of dissection in cases of lung cancer presenting with pure ground-glass nodule and mediastinal lymph node dissection in cases of solid tumors and tumors with solid lesions and ground-glass nodule. The procedure was classified into 1 of 3 groups (level 1,2, or 3) according to the previously reported difficulty classification of the segmentectomy. ${ }^{7}$ Level 1 is easy, for example, superior and lingular segmentectomy: a single intersegmental dissection surface or similar cases of thoracoscopic surgery. Level 2 is fairly difficult, for example, apical, anterior, and posterior segmentectomy: multiple dissection surfaces that contact at obtuse angles or instances where there are no similar reported cases of thoracoscopic surgery even with a single dissection surface. The

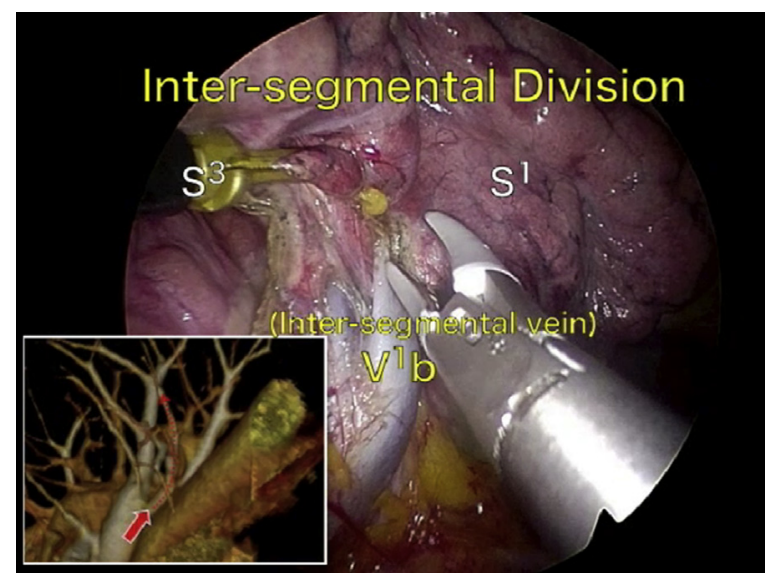

VIDEO 1. Port-access thoracoscopic anatomic right anterior segmentectomy. Video available at: https://www.jtcvs.org/article/S0022-5223(18)31835-X/ fulltext.

representative procedure (right anterior segmentectomy; level 2) is presented in Video 1. Level 3 is difficult, for example, lateral and posterior basal segmentectomy: multiple dissection surfaces at acute angles or a bronchial pulmonary artery located deep within the segment. The Clavien-Dindo classification was used for the assessment of postoperative complications. ${ }^{12}$ Data collection and analyses were approved (Institutional Review Board No. 2017-484), and the need for written informed consent from each patient was waived by the Institutional Review Board.

\section{Cumulative Sum Analysis}

The learning curve was analyzed using the cumulative sum (CUSUM) method similar to that described by Bokhari and colleagues ${ }^{13}$ and Yamaguchi and colleagues. ${ }^{14}$ The CUSUM is the running total of the differences between the individual data points and the mean of all data points. Thus, CUSUM can be performed recursively. The learning curve was evaluated using OT and the cumulative sum value of OT (CUSUM $\left.{ }_{\mathrm{OT}}\right)$ in all cases, in each group, and regarding just the leading surgeon at our institution. First, the cases were ordered chronologically from the earliest to the latest date of the surgery. The CUSUM $\mathrm{OT}_{\mathrm{OT}}$ of the first case was the difference between the OT for the first case and the mean OT for all cases. The CUSU$\mathrm{M}_{\mathrm{OT}}$ of the second case was the CUSUM $\mathrm{OT}_{\mathrm{OT}}$ of the previous case added to the difference between the OT for the second case and the mean OT for all cases. This recursive process continued until CUSUM OT $_{\text {for the last }}$ case was calculated as zero. We performed the curve of best fit for the plot for detecting a change in slope of CUSUM $_{\mathrm{OT}}$ learning curve. The required cases were calculated from the inflection point of the curve of the line representing the best fit for the plot.

\section{Statistical Analysis}

Statistical analysis was performed using JMP software, version 11.0 (SAS Institute Inc, Cary, NC). For comparisons of variances between curve phases or between operation groups, the Wilcoxon rank-sum test or the chisquare test was used throughout unless otherwise specified.

\section{RESULTS}

During the study period, 252 patients underwent VATS segmentectomy. Patient demographics, surgical procedures, intraoperative characteristics, short-term postoperative outcomes, and pathologic diagnoses are summarized in Table 1 . The series comprised 121 male patients and $131 \mathrm{fe}$ male patients, with a mean age of 66 years. Surgery was 
TABLE 1. Demographic and clinical characteristics of the patients

\begin{tabular}{|c|c|c|c|c|}
\hline Characteristics & $\begin{array}{l}\text { All cases } \\
(\mathbf{n}=\mathbf{2 5 2})\end{array}$ & $\begin{array}{c}\text { Leading } \\
\text { surgeon }(A)(n=153)\end{array}$ & $\begin{array}{c}\text { Nonleading } \\
\text { surgeon }(\mathrm{B})(\mathrm{n}=\mathbf{9 9})\end{array}$ & $\begin{array}{l}(A) \text { vs }(B) \\
(P \text { value })\end{array}$ \\
\hline \multicolumn{5}{|l|}{ Sex, n $(\%)$} \\
\hline Male & $121(48.0)$ & $75(49.0)$ & $46(46.5)$ & \\
\hline Female & $131(52.0)$ & $78(51.0)$ & $53(53.5)$ & .789 \\
\hline \multicolumn{5}{|l|}{ Age } \\
\hline Median (IQR), y & $68(61-75)$ & $68(61-76)$ & $69(59-75)$ & .963 \\
\hline$<50 \mathrm{y}, \mathrm{n}(\%)$ & $22(8.7)$ & $15(9.8)$ & $7(7.1)$ & \\
\hline $50-<65 \mathrm{y}, \mathrm{n}(\%)$ & $77(30.6)$ & $46(30.1)$ & $31(31.3)$ & \\
\hline$>65 \mathrm{y}, \mathrm{n}(\%)$ & $153(60.7)$ & $92(60.1)$ & $61(61.6)$ & .799 \\
\hline \multicolumn{5}{|l|}{ ASA score } \\
\hline Median (IQR) & $2(1-2)$ & $2(1-2)$ & $2(1-2)$ & .837 \\
\hline \multicolumn{5}{|l|}{ Comorbidity index } \\
\hline Median (IQR) & $0(0-1)$ & $0(0-1)$ & $0(0-1)$ & .174 \\
\hline Surgical side (right/left) & $134 / 118$ & $86 / 67$ & $48 / 51$ & .247 \\
\hline \multicolumn{5}{|l|}{ Location, $\mathrm{n}(\%)$} \\
\hline RUL & $72(28.6)$ & $41(26.8)$ & $31(31.2)$ & \\
\hline RML & $5(2.0)$ & $3(2.0)$ & $2(2.0)$ & \\
\hline RLL & $58(23.0)$ & $42(27.5)$ & $16(16.2)$ & \\
\hline LUL & $77(30.5)$ & $42(27.5)$ & $35(35.4)$ & \\
\hline LLL & $40(15.9)$ & $25(16.2)$ & $15(15.2)$ & .262 \\
\hline \multicolumn{5}{|l|}{ Surgical level, n (\%) } \\
\hline Level 1 & $60(23.8)$ & $24(15.6)$ & $36(36.4)$ & \\
\hline Level 2 & $145(57.5)$ & $85(55.6)$ & $60(60.6)$ & \\
\hline Level 3 & 47 (18.7) & $44(28.8)$ & $3(3.0)$ & $<.001$ \\
\hline \multicolumn{5}{|l|}{ Nodal dissection, n (\%) } \\
\hline Mediastinal & $34(13.5)$ & $22(14.4)$ & $12(12.1)$ & .707 \\
\hline \multicolumn{5}{|l|}{ OT } \\
\hline Mean (SD), min & $188.4(61.1)$ & $182.3(63.3)$ & $197.8(56.6)$ & $.049^{*}$ \\
\hline \multicolumn{5}{|l|}{ Bleeding } \\
\hline Median (IQR), mL & $50(5-129)$ & $53(5-132)$ & $50(5-128)$ & .769 \\
\hline Conversion to open thoracotomy due to bleeding, $\mathrm{n}(\%)$ & $5(2.0)$ & $2(1.3)$ & $3(3.0)$ & .384 \\
\hline \multicolumn{5}{|l|}{ Drainage } \\
\hline Median (IQR), d & $1(1-2)$ & $1(1-2)$ & $1(1-1)$ & .087 \\
\hline \multicolumn{5}{|l|}{ Length of hospital stay } \\
\hline Median (IQR), POD & $6(5-9)$ & $7(5-9)$ & $6(5-8)$ & .709 \\
\hline Complication, $\mathrm{n}(\%)$ & $30(11.9)$ & $21(13.7)$ & $9(9.1)$ & .322 \\
\hline \multicolumn{5}{|l|}{ Clavien-Dindo classification } \\
\hline Greater than grade III, n (\%) & $20(7.9)$ & $17(11.1)$ & $3(3.0)$ & .029 \\
\hline Postoperative bleeding, $\mathrm{n}(\%)$ & $2(0.8)$ & $1(0.7)$ & $1(1.0)$ & 1 \\
\hline 30-d mortality rate, $\mathrm{n}(\%)$ & $1(0.4)$ & $1(0.7)$ & $0(0)$ & 1 \\
\hline 30-d readmission rate, $\mathrm{n}(\%)$ & $4(1.6)$ & $2(1.3)$ & $2(2.0)$ & .647 \\
\hline \multicolumn{5}{|l|}{ Pathologic diagnosis, $\mathrm{n}(\%)$} \\
\hline Lung cancer & $191(75.8)$ & $109(71.2)$ & $82(82.8)$ & \\
\hline Metastasis & 40 (15.9) & $29(19.0)$ & $11(11.1)$ & \\
\hline Benign & $21(8.3)$ & $15(9.8)$ & $6(6.1)$ & .112 \\
\hline
\end{tabular}

$I Q R$, Interquartile range, $A S A$, American Society of Anesthesiologists; $R U L$, right upper lobe; $R M L$, right middle lobe; $R L L$, right lower lobe; $L U L$, left upper lobe; $L L L$, left lower lobe; $O T$, operative time; $S D$, standard deviation; $P O D$, postoperative day. *Student $t$ test.

performed for lung cancers in 191 cases, metastases in 40 cases, and benign diseases in 21 cases. The leading surgeon performed 153 cases. Twelve surgeons performed the other
99 cases. Of these, 9 surgeons were residents before acquiring a certification of specialist for general thoracic surgery in Japan. The second most surgeries performed included 


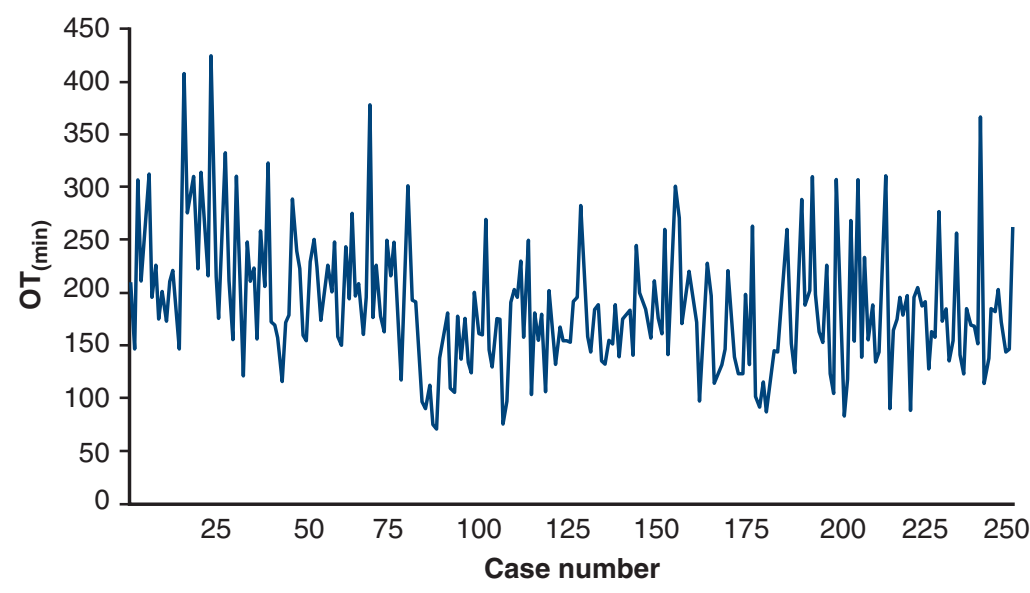

FIGURE 1. The raw OT times were plotted in chronologic case order. OT, Operative time.

38 cases, and the third most surgeries performed included 28 cases. The other surgeons performed fewer surgeries. Comparing the patient characteristics of the leading surgeon and the nonleading surgeon, there were significant differences in surgical location, level, OT, and complication. The leading surgeon had significantly more level 3 segmentectomies than the nonleading surgeons $(P<.001)$. The mean OT was 188 minutes, and the mean amount of bleeding was $92 \mathrm{~mL}$. Among the 252 patients, 60 were level 1 (easy), 145 were level 2 (fairly difficult), and 47 were level 3 (difficult) cases. Mediastinal and hilar lymphadenectomy was performed in 34 cases $(13.5 \%)$. There was no significant difference between phase 1 and 2 compared with phase 3 . Also, there was no significant difference between the leading surgeon and the nonleading surgeon. Conversion to open thoracotomy was performed in 5 patients $(2.0 \%)$, all of which were due to intraoperative bleeding. Postoperative complications occurred in 30 patients $(11.9 \%)$, including 19 air leakages, 5 pneumonias, 2 bleeds, and others. Hospital mortality and mortality within 30 days was observed in 1 patient $(0.4 \%)$ because of acute respiratory distress syndrome, and 30-day readmission was observed in 4 patients $(1.6 \%)$.

The raw OT times were plotted in chronologic case order (Figure 1). The OT times gradually decreased, but the trend is unclear. The CUSUM ${ }_{\mathrm{OT}}$ learning curve was best modeled as a second-order polynomial (parabola) with the CUSUM $_{\mathrm{OT}}$ equation in minutes equal to $0.09 \times$ case number ${ }^{2}-15.14 \times$ case number -1087.26 (Figure 2). Once we applied this method, we obtained 3 well-differentiated phases. Phase 1 (the initial 61 cases) represented the initial learning phase. Phase 2 (the middle 23 cases) represented increased competence in the VATS segmentectomy. Phase 3 (the final 168 cases) represented the period of highest skill or mastery of the

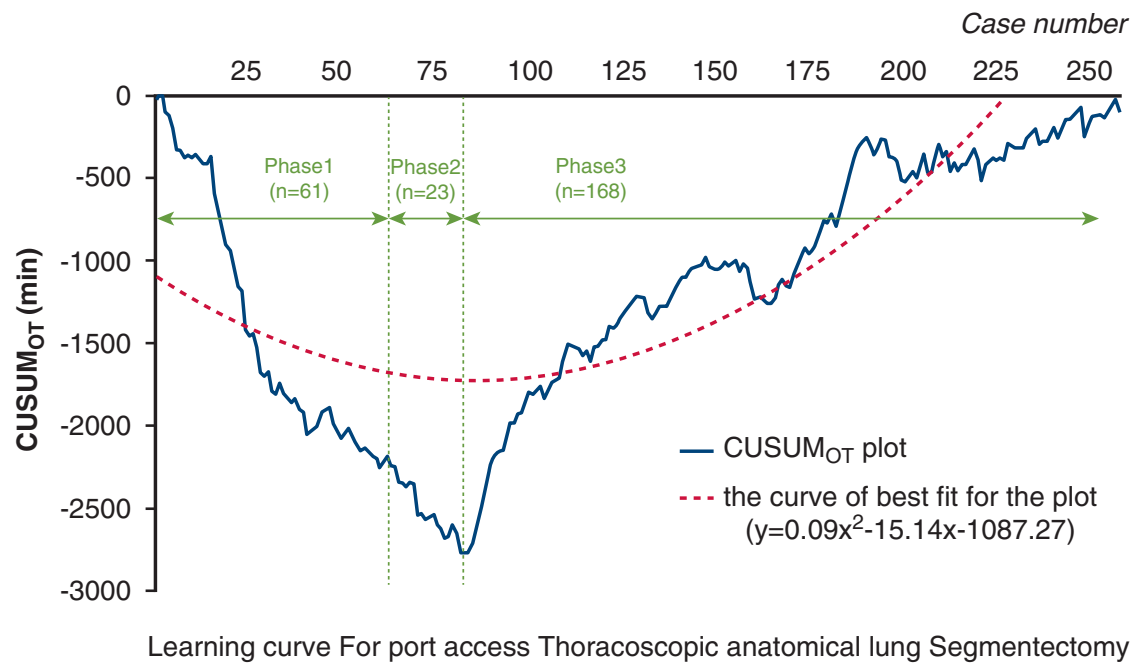

FIGURE 2. CUSUM ${ }_{\mathrm{OT}}$ in all cases plotted against case number (solid line). The dashed line represents the curve of best fit for the plot (a second-order

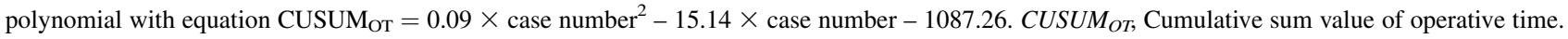


TABLE 2. Interphase comparisons of patient characteristics and operative parameters in all cases

\begin{tabular}{|c|c|c|c|c|}
\hline Characteristics & Phase $1(n=61)$ & Phase $2(n=23)$ & Phase $3(n=168)$ & $\begin{array}{l}\text { Phase } 1 \text { and } 2 \text { vs } \\
\text { phase } 3 \text { ( } P \text { value) }\end{array}$ \\
\hline \multicolumn{5}{|l|}{ Sex, n $(\%)$} \\
\hline Male & $29(47.5)$ & $10(43.5)$ & $82(48.8)$ & .789 \\
\hline \multicolumn{5}{|l|}{ Age } \\
\hline Median (IQR), y & $68(59-76)$ & $66(58-77)$ & $69(62-75)$ & .715 \\
\hline \multicolumn{5}{|l|}{ ASA score } \\
\hline Median (IQR) & $2(1-2)$ & $2(1-2)$ & $2(1-2)$ & .048 \\
\hline \multicolumn{5}{|l|}{ Comorbidity index } \\
\hline Median (IQR) & $0(0-1)$ & $0(0-1)$ & $0(0-1)$ & .593 \\
\hline Surgical side right, $\mathrm{n}(\%)$ & $33(54.1)$ & $12(52.2)$ & $89(53.0)$ & 1 \\
\hline \multicolumn{5}{|l|}{ Location, $\mathrm{n}(\%)$} \\
\hline RUL & $15(24.6)$ & $9(39.1)$ & $48(28.6)$ & \\
\hline RML & $1(1.6)$ & $0(0)$ & $4(2.4)$ & \\
\hline RLL & $17(27.9)$ & $3(13.0)$ & $38(22.6)$ & \\
\hline LUL & $18(29.5)$ & $7(30.5)$ & $52(31.0)$ & \\
\hline LLL & $10(16.4)$ & $4(17.4)$ & $26(15.5)$ & .992 \\
\hline \multicolumn{5}{|l|}{ Surgical level, n (\%) } \\
\hline Level 1 & $19(31.1)$ & $5(21.7)$ & $36(21.4)$ & \\
\hline Level 2 & $31(50.8)$ & $12(52.2)$ & $102(60.7)$ & \\
\hline Level 3 & $11(18.1)$ & $6(26.1)$ & $30(17.9)$ & .329 \\
\hline \multicolumn{5}{|l|}{ Nodal dissection, $\mathrm{n}(\%)$} \\
\hline Mediastinal & $12(19.7)$ & $2(8.7)$ & $20(11.9)$ & .331 \\
\hline \multicolumn{5}{|l|}{ OT } \\
\hline Mean (SD), min & $223.9(64.6)$ & $207.4(61.0)$ & $172.9(53.6)$ & $<.001 *$ \\
\hline \multicolumn{5}{|l|}{ Bleeding } \\
\hline Median (IQR), mL & $100(10-163)$ & $146(57-213)$ & $27(3-89)$ & $<.001$ \\
\hline Conversion to open thoracotomy due to bleeding, $\mathrm{n}(\%)$ & $3(4.9)$ & $0(0)$ & $2(1.2)$ & .337 \\
\hline \multicolumn{5}{|l|}{ Drainage } \\
\hline Median (IQR), d & $1(1-2)$ & $1(1-1)$ & $1(1-1)$ & .239 \\
\hline \multicolumn{5}{|l|}{ Length of hospital stay } \\
\hline Median (IQR), POD & $7(5-11)$ & $6(5-9)$ & $6(5-8)$ & .053 \\
\hline Complication, $\mathrm{n}(\%)$ & $12(19.7)$ & $1(4.3)$ & $17(10.1)$ & .222 \\
\hline \multicolumn{5}{|l|}{ Clavien-Dindo classification } \\
\hline Greater than grade III, $\mathrm{n}(\%)$ & $8(13.1)$ & $0(0)$ & $12(7.1)$ & .622 \\
\hline Postoperative bleeding, $\mathrm{n}(\%)$ & $0(0)$ & $0(0)$ & $2(1.2)$ & .554 \\
\hline 30-d mortality rate, $\mathrm{n}(\%)$ & $1(1.6)$ & $0(0)$ & $0(0)$ & .333 \\
\hline 30-d readmission rate, $\mathrm{n}(\%)$ & $0(0)$ & $0(0)$ & $4(2.4)$ & .304 \\
\hline \multicolumn{5}{|l|}{ Pathologic diagnosis, $\mathrm{n}(\%)$} \\
\hline Lung cancer & $46(75.4)$ & $17(73.9)$ & $128(76.2)$ & \\
\hline Metastasis & $10(16.4)$ & $4(17.4)$ & $26(15.5)$ & \\
\hline Benign & $5(8.2)$ & $2(8.7)$ & $14(8.3)$ & .972 \\
\hline
\end{tabular}

$I Q R$, Interquartile range, $A S A$, American Society of Anesthesiologists; $R U L$, right upper lobe; $R M L$, right middle lobe; $R L L$, right lower lobe; $L U L$, left upper lobe; $L L L$, left lower lobe; $O T$, operative time; $S D$, standard deviation; $P O D$, postoperative day. *Student $t$ test.

VATS segmentectomy procedure. The inflection point occurred after 84 cases. If the learning phases are considered as the initial learning phase and the increased competence phase, this inflection point is the boundary line between the learning phase and the competent phase.
Because the phase 2 line does not show a perfect plateau, the near-plateau point is the boundary line between phase 1 and phase 2. The inflection point was at 61 cases.

Comparisons of various parameters among the 3 phases identified by CUSUM ${ }_{\mathrm{OT}}$ analysis are presented in Table 2 . 
Age, sex, comorbidity index, surgical side, surgical level, location, and pathologic diagnosis did not differ significantly among the 3 phases. The ASA differed significantly in phase 3 compared with phase 1 and phase 2 combined $(P=.048)$.

Interphase comparisons of the intraoperative parameters (OT, bleeding, conversion to open thoracotomy due to bleeding) and postoperative parameters (drainage, LOS, complications, Clavien-Dindo grade $>$ III, and postoperative bleeding) are presented in Table 2. Significant reductions in OT and bleeding were observed between phase 1 and 2 compared with phase $3(P<.001$ and $P<.001$, respectively). Therefore, there was a significant reduction in the OT and bleeding in phase 3. Significant reductions in conversion to open thoracotomy due to bleeding were not observed between phase 1 and 2 compared with phase 3 , but there was less of a trend for conversion to open thoracotomy due to bleeding in phase 3 .

Comparing the patient characteristics of the leading surgeon and nonleading surgeons, there was a significant difference in level 3 segmentectomy. Therefore, we examined the learning curves of the leading surgeon and nonleading surgeons, excluding level 3 segmentectomy. The CUSUM $_{\mathrm{OT}}$ learning curve for the leading surgeon was best modeled as a second-order polynomial (parabola) with the CUSUM $_{\mathrm{OT}}$ equation in minutes equal to $0.15 \times$ case number ${ }^{2}-9.45 \times$ case number -671.46 (Figure 3). Phase 1 occurred during the initial 18 cases, phase 2 occurred during the middle 14 cases, and phase 3 occurred during the final 77 cases. Comparisons of operative parameters among the 3 phases identified by CUSU$\mathrm{M}_{\mathrm{OT}}$ analysis are presented in Table 3. Significant reductions in OT and bleeding were observed between phase 1 and 2 compared with phase $3(P<.001$ and .002 , respectively). The CUSUM ${ }_{\mathrm{OT}}$ learning curve for the nonleading surgeon was best modeled as a second-order polynomial (parabola) with the CUSUM ${ }_{\mathrm{OT}}$ equation in minutes equal to $0.25 \times$ case number ${ }^{2}-18.99 \times$ case number - 194.05 (Figure 4). Phase 1 occurred during the initial 28 cases, phase 2 occurred during the middle 10 cases, and phase 3 occurred during the final 58 cases. Comparisons of operative parameters between the 3 phases identified by CUSUM ${ }_{\mathrm{OT}}$ analysis are presented in Table 3 . Significant reductions in OT and LOS were observed between phase 1 and 2 compared with phase $3(P=.019$ and .011$)$.

\section{DISCUSSION}

The learning curve is a graphical representation of the temporal relationship between the surgeon's mastery of a specifically assigned task and the chronologic number of cases performed. The CUSUM technique is a method adopted by the medical profession in the 1970s to analyze the learning curve for surgical procedures. ${ }^{15,16}$ We have safely performed the segmentectomy procedure in more than 250 cases at our institution. During this period, all of the conversions to open thoracotomy were due to bleeding. Both cases of postoperative bleeding were ones involving left upper division segmentectomy VATS with mediastinal lymph node dissection, one of which required hemostasis because of bleeding from the bronchial artery, and the other one improved without intervention. Previously, we reported the use of 3D CT images for surgical simulation during an anatomic thoracoscopic pulmonary segmentectomy and the effectiveness of this technique. ${ }^{6,7,11} \mathrm{We}$ also previously reported the posterior approach to a thoracoscopic pulmonary segmentectomy of a dorsal basal segment ${ }^{10}$ and a thoracoscopic anatomic lung segmentectomy using 3D CT simulation without tumor markings for nonpalpable and nonvisualized small lung nodules. ${ }^{11}$ This enabled the resection of smaller areas, such as subsegments. The learning curve for VATS lobectomy has been reported ${ }^{17,18}$; however, the learning curve

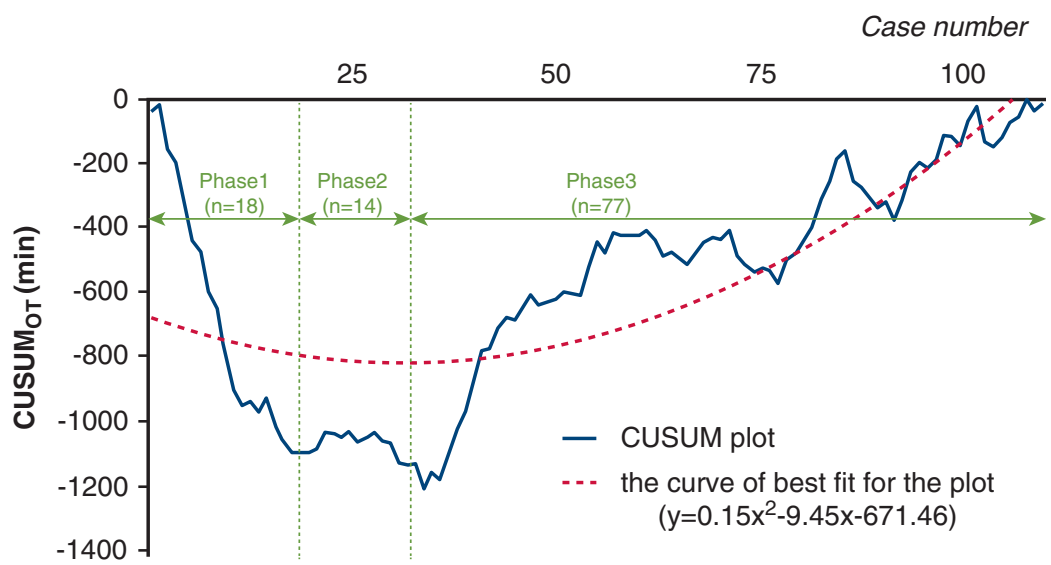

FIGURE 3. CUSUM ${ }_{\mathrm{OT}}$ for the leading surgeon excluding level 3 segmentectomy plotted against case number (solid line). The dashed line represents the curve of best fit for the plot (a second-order polynomial with equation CUSUM $_{\mathrm{OT}}=0.15 \times$ case number $2-9.45 \times$ case number -671.46 . CUSUM OT $_{\text {, }}$ Cumulative sum value of operative time. 
TABLE 3. Interphase comparisons of operative parameters between the leading and nonleading surgeons excluding level 3 segmentectomy

\begin{tabular}{|c|c|c|c|c|c|c|c|c|}
\hline \multirow[b]{2}{*}{ Characteristics } & \multicolumn{3}{|c|}{ Leading surgeon } & \multirow[b]{2}{*}{$\begin{array}{l}\text { Phase } 1 \text { and } 2 \text { vs } \\
\text { Phase } 3 \text { ( } P \text { value) }\end{array}$} & \multicolumn{3}{|c|}{ Nonleading surgeons } & \multirow[b]{2}{*}{$\begin{array}{l}\text { Phase } 1 \text { and } 2 \text { vs } \\
\text { phase } 3 \text { ( } P \text { value) }\end{array}$} \\
\hline & $\begin{array}{l}\text { Phase 1 } \\
(n=18)\end{array}$ & $\begin{array}{l}\text { Phase } 2 \\
(n=14)\end{array}$ & $\begin{array}{l}\text { Phase 3 } \\
(n=77)\end{array}$ & & $\begin{array}{l}\text { Phase 1 } \\
(n=28)\end{array}$ & $\begin{array}{l}\text { Phase 2 } \\
(\mathbf{n}=10)\end{array}$ & $\begin{array}{l}\text { Phase 3 } \\
(n=58)\end{array}$ & \\
\hline $\begin{array}{l}\text { OT } \\
\quad \text { Mean (SD), min }\end{array}$ & $\begin{array}{l}230.1 \\
(57.1)\end{array}$ & $\begin{array}{l}171.9 \\
(26.5)\end{array}$ & $\begin{array}{l}154.4 \\
(46.0)\end{array}$ & $<.001 *$ & $\begin{array}{l}228.6 \\
(61.3)\end{array}$ & $\begin{array}{l}173.0 \\
(45.6)\end{array}$ & $\begin{array}{l}186.1 \\
(51.3)\end{array}$ & $.019^{*}$ \\
\hline $\begin{array}{l}\text { Bleeding } \\
\quad \text { Median (IQR), mL }\end{array}$ & $\begin{array}{c}92 \\
(42-116)\end{array}$ & $\begin{array}{c}57 \\
(34-13)\end{array}$ & $\begin{array}{r}20 \\
(3-75)\end{array}$ & .002 & $\begin{array}{c}111 \\
(10-187)\end{array}$ & $\begin{array}{r}31 \\
(1-54)\end{array}$ & $\begin{array}{r}38 \\
(4-95)\end{array}$ & .053 \\
\hline $\begin{array}{l}\text { Conversion to open } \\
\text { thoracotomy due to } \\
\text { bleeding, } \mathrm{n}(\%)\end{array}$ & $1(5.6)$ & $0(0)$ & $1(1.3)$ & .503 & $2(7.1)$ & $0(0)$ & $1(1.7)$ & .561 \\
\hline $\begin{array}{l}\text { Drainage } \\
\quad \text { Median (IQR), d }\end{array}$ & $1(1-5)$ & $1(1-2)$ & $1(1-2)$ & .171 & $1(1-2)$ & $1(1-1)$ & $1(1-1)$ & .690 \\
\hline $\begin{array}{l}\text { Length of hospital stay } \\
\text { Median (IQR), POD } \\
\text { Complication, n (\%) }\end{array}$ & $\begin{array}{r}11(6-14) \\
3(16.7)\end{array}$ & $\begin{array}{l}6(5-7) \\
3(21.4)\end{array}$ & $\begin{array}{c}7(5-8) \\
12(15.6)\end{array}$ & $\begin{array}{l}.258 \\
.778\end{array}$ & $\begin{array}{l}8(5-10) \\
5(17.9)\end{array}$ & $\begin{array}{l}7(6-11) \\
1(10.0)\end{array}$ & $\begin{array}{l}6(5-8) \\
3(5.2)\end{array}$ & $\begin{array}{l}.011 \\
.148\end{array}$ \\
\hline $\begin{array}{l}\text { Clavien-Dindo classification } \\
\text { Greater than grade III, } \\
\text { n (\%) }\end{array}$ & $2(11.1)$ & $3(21.4)$ & $10(13.0)$ & .763 & $2(7.1)$ & $0(0)$ & $1(1.7)$ & .560 \\
\hline Postoperative bleeding, n (\%) & $0(0)$ & $0(0)$ & $1(1.3)$ & 1 & $0(0)$ & $0(0)$ & $1(1.7)$ & 1 \\
\hline
\end{tabular}

$O T$, Operative time; $S D$, standard deviation; $I Q R$, interquartile range; $P O D$, postoperative day. *Student $t$ test.

for VATS segmentectomy has not yet been reported. Therefore, we used the CUSUM method to investigate the learning curve for VATS segmentectomy for benign and malignant lung diseases. During the past year, several reports have been published that argue for monitoring on an individual case-after-case fashion (sequential monitoring) using the ever-expanding suite of statistical quality-control techniques. The most commonly used statistical quality-control techniques belong to the family of CUSUM charts. These charts purport to provide early identification of deviation from a performance standard. ${ }^{19}$ The CUSUM analysis was often used to analyze the surgical time data by visually inspecting the plots for robotic surgery and VATS. Recently, some methods using CUSUM to evaluate surgical outcomes were reported..$^{20,21}$ The CUSUM method described by Bokhari and colleagues ${ }^{13}$ and Yamaguchi and colleagues ${ }^{14}$ was useful for recognizing the graphics with 3 well-differentiated phases. Therefore, we chose their method for our study. The focus of this study was on investigating OT as a surrogate marker for operative competency by dividing it into phases shown to correlate with process components of surgeon learning.

This study reports the learning curve for all cases, for each group (level 1,2,3) according to the previously

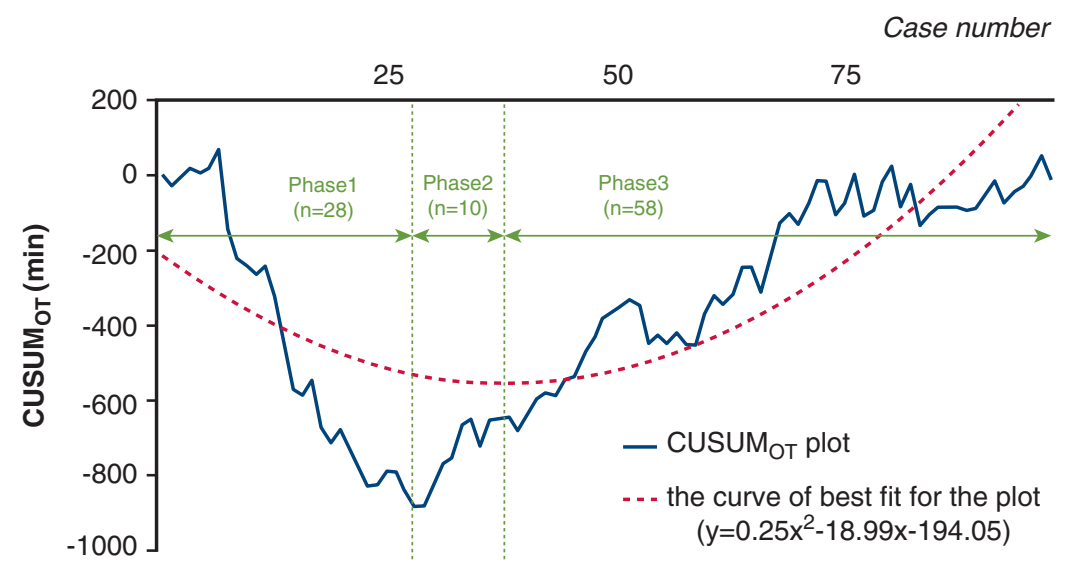

FIGURE 4. CUSUM $_{\mathrm{OT}}$ for nonleading surgeon excluding level 3 segmentectomy plotted against case number (solid line). The dashed line represents the curve of best fit for the plot (a second-order polynomial with equation CUSUM $_{\mathrm{OT}}=0.25 \times$ case number $2-18.99 \times$ case number -194.05 . CUSUM $O$, Cumulative sum value of operative time. 
reported difficulty classification for a segmentectomy, ${ }^{7}$ as well as for the leading surgeon at our institution. We chose CUSUM analysis because meaningful conclusions cannot be drawn from raw data plotted in chronologic order (Figure 1). The CUSUM ${ }_{\mathrm{OT}}$ graph (Figure 2) shows the variance from the mean on a case-by-case basis, yielding a parabolic curve with 3 distinct phases from which correlates of the learning curve can be assessed. The negative slope in phase 1 indicates shorter OTs during this learning curve phase (with respect to the mean OT for all cases). The positive slope in phases 2 and 3 indicates longer OTs (with respect to the mean OT for all cases), which are necessary for the performance of more complicated cases with increased surgical competence.

In all cases, phase 1 represents the initial portion of the learning curve, found to include 61 cases. An additional 23 cases comprise phase 2 , which represents the accumulation of additional experience once the initial phase of the learning curve has been passed. As we compared a combination of phase 1 and phase 2 with phase 3 , we observed significant differences in relation to the ASA; however, we did not observe significant differences in relation to comorbidities and other parameters (Table 2). Interphase comparisons of intraoperative parameters and short-term outcomes are presented in Table 2. A significant reduction in the OT and bleeding was observed between phase 1 and 2 compared with phase $3(P<.001$ and $P<.001$, respectively). The number of complications was slightly lower in phase 1; however, a significant reduction in complications was not observed.

Regarding the leading surgeon, the learning curve and interphase comparisons of the operative parameters and short-term outcomes showed almost similar results (Figure 3, Table 3). Comparing the patient characteristics of the leading surgeon and nonleading surgeons, there was a significant difference in level 3 segmentectomy. Therefore, we examined the learning curves of the leading surgeon and nonleading surgeons excluding level 3 . The leading surgeon needed 32 cases and the nonleading surgeons needed 38 cases, not including level 3 segmentectomy. In our institution, we perform operations as one team and the leading surgeon guides the second surgeon. After the second surgeon assisted several times, he or she becomes the operator. Therefore, the leading surgeon may have attained experience faster or have been transmitted the information about the procedure. There are various kinds of segmentectomies; therefore, stratification of difficulty should be considered. The mean OTs of levels 1, 2, and 3 were 182, 182, and 215 minutes, respectively. According to the difficulty classification of segmentectomies, we also observed a significant reduction in OT after 25 (level 1), 49 (level 2), and 19 (level 3) cases. As for the leading surgeon, we observed a significant reduction in OT after 53 (all cases), 11 (level 1), 22 (level 2), and 19 (level 3) cases. This result shows that the performance of level $3 \mathrm{seg}$ mentectomies needed a smaller number of cases compared with level 1 or level 2 segmentectomies. Because the level 3 segmentectomy is a more difficult procedure, it was not performed in the initial phase of this procedure.

\section{Study Limitations}

A limitation of this study is its retrospective nature and that it was conducted in a single institute. As also indicated by Gonfiotti and colleagues, ${ }^{22}$ several factors can affect the duration and efficacy of the learning curve period; the experience in other complex VATS procedures or in standard open major pulmonary resections; the selection of a dedicated surgical team; the opportunity to concentrate on the learning curve procedures within a short period; and finally, the development of a proctored and stepwise program. Although this study was divided by surgical level, the difficulty of the procedure differs, depending on anatomic variations among each level. Because there was a significant difference in level 3 segmentectomy when comparing the patient characteristics of the leading and nonleading surgeons, we could not compare the learning curves in all of the segmentectomies including level 3 . Also, we could not compare the margin status because it covered not only lung cancer but also benign disease. Complete resection of tumors was attempted by performing segmentectomy for tumors located between intersegmental veins on a preoperative 3D CT simulation. Moreover, when it was assumed that simple segmentectomy alone would yield inadequate surgical margins $(<1 \mathrm{~cm})$ or if a margin-to-tumor diameter ratio less than 1 was anticipated, segmentectomy combined with adjacent subsegmentectomy was planned.

\section{CONCLUSIONS}

The 3 phases identified with the CUSUM analysis of the OT represented the characteristic stages of the learning curve for VATS segmentectomy. The data suggest that the inflection point for the learning curve was achieved after 84 cases in our institution. The leading surgeon needed 32 cases and the nonleading surgeons needed 38 cases (excluding level 3 segmentectomy) to achieve this level of competence.

\section{Conflict of Interest Statement}

Authors have nothing to disclose with regard to commercial support.

\section{References}

1. McKenna R Jr. VATS lobectomy with mediastinal lymph node sampling or dissection. Chest Surg Clin N Am. 1995;5:223-32.

2. Onaitis MW, Petersen RP, Balderson SS, Toloza E, Burfeind WR, Harpole DH, et al. Thoracoscopic lobectomy is a safe and versatile procedure: experience with 500 consecutive patients. Ann Surg. 2006;244:420-5.

3. Swanson SJ, Henderson JE II, D’Amico TA, Demmy TL, McKenna RJ Jr, Green MR, et al. Video-assisted thoracic surgery lobectomy: report of CALGB 
39802. A prospective, multi-institution feasibility study. J Clin Oncol. 2007;25: 4993-7.

4. Whitson BA, Andrade RS, Boettcher A, Bardales R, Kratzke RA, Dahlberg PS, et al. Video-assisted thoracoscopic surgery is more favorable than thoracotomy for resection of clinical stage I non-small cell lung cancer. Ann Thorac Surg. 2007:83:1965-70.

5. Watanabe A, Koyanagi T, Ohsawa H, Mawatari T, Nakashima S, Takahashi N, et al. Systematic node dissection by VATS is not inferior to that through an open thoracotomy: a comparative clinicopathologic retrospective study. Surgery. 2005;138:510-7.

6. Oizumi H, Kanauchi N, Kato H, Endoh M, Takeda S, Suzuki J, et al. Total thoracoscopic pulmonary segmentectomy. Eur J Cardiothorac Surg. 2009;36:374-7.

7. Oizumi H, Kanauchi N, Kato H, Endoh M, Takeda S, Suzuki J, et al. Anatomic thoracoscopic pulmonary segmentectomy under 3-dimensional multidetector computed tomography simulation: a report of 52 consecutive cases. J Thorac Cardiovasc Surg. 2011;141:678-82.

8. Oizumi H, Kato H, Endoh M, Inoue T, Watarai H, Sadahiro M. Slip knot bronchial ligation method for thoracoscopic lung segmentectomy. Ann Thorac Surg. 2014;97:1456-8

9. Oizumi H, Kato H, Endoh M, Suzuki J, Watarai H, Suzuki K, et al. Management of bronchial stumps in anatomic lung segmentectomy. Ann Thorac Surg. 2016; 101:2120-4.

10. Endo M, Oizumi H, Kato H, Suzuki J, Watarai H, Masaoka T, et al. Posterior approach to thoracoscopic pulmonary segmentectomy of the dorsal basal segment: a single-institute retrospective review. J Thorac Cardiovasc Surg. 2017; $154: 1432-9$.

11. Kato H, Oizumi H, Suzuki J, Hamada A, Watarai H, Sadahiro M. Thoracoscopic anatomical lung segmentectomy using 3D computed tomography simulation without tumour markings for non-palpable and non-visualized small lung nodules. Interact Cardiovasc Thorac Surg. 2017;25:434-41.

12. Clavien PA, Strasberg SM. Severity grading of surgical complications. Ann Surg. 2009:250:197-8.
13. Bokhari MB, Patel CB, Ramos-Valadez DI, Ragupathi M, Haas EM. Learning curve for robotic-assisted laparoscopic colorectal surgery. Surg Endosc. 2011;25:855-60.

14. Yamaguchi T, Kinugasa Y, Shiomi A, Sato S, Yamakawa Y, Kagawa H, et al Learning curve for robotic-assisted surgery for rectal cancer: use of the cumulative sum method. Surg Endosc. 2015;29:1679-85.

15. Chaput de Saintonge DM, Vere DW. Why don't doctors use CUSUMs? Lancet 1974;1:120-1.

16. Wohl H. The CUSUM plot: its utility in the analysis of clinical data. $N$ Engl J Med. 1977;296:1044-5.

17. Belgers EH, Siebenga J, Bosch AM, van Haren EH, Bollen EC. Complete videoassisted thoracoscopic surgery lobectomy and its learning curve. A single center study introducing the technique in The Netherlands. Interact Cardiovasc Thorac Surg. 2010;10:176-80.

18. Petersen RH, Hansen HJ. Learning thoracoscopic lobectomy. Eur J Cardiothorac Surg. 2010;37:516-20

19. Blackstone EH. Monitoring surgical performance. J Thorac Cardiovasc Surg 2004; 128:807-10.

20. Zhang H, Chen L, Wang Z, Zheng Y, Geng Y, Wang F, et al. The learning curve for robotic McKeown esophagectomy in patients with esophageal cancer. Ann Thorac Surg. 2018;105:1024-30.

21. Li X, Wang J, Ferguson MK. Competence versus mastery: the time course for developing proficiency in video-assisted thoracoscopic lobectomy. J Thorac Cardiovasc Surg. 2014;147:1150-4.

22. Gonfiotti A, Bongiolatti S, Borgianni S, Borrelli R, Jaus MO, Politi L, et al Development of a video-assisted thoracoscopic lobectomy program in a single institution: results before and after completion of the learning curve. J Cardiothorac Surg. 2016;11:130.

Key Words: thoracoscopy/video-assisted thoracic surgery (VATS), segmentectomy/subegmentectomy, learning curve/3D reconstruction 